\title{
On Dynamical Behavior of Discrete Time Fuzzy Logistic Equation
}

\author{
Qianhong Zhang $(\mathbb{D}$ and Fubiao Lin \\ School of Mathematics and Statistics, Guizhou University of Finance and Economics, Guiyang, Guizhou 550025, China \\ Correspondence should be addressed to Qianhong Zhang; zqianhong68@163.com
}

Received 15 August 2018; Accepted 29 November 2018; Published 11 December 2018

Academic Editor: Douglas R. Anderson

Copyright (c) 2018 Qianhong Zhang and Fubiao Lin. This is an open access article distributed under the Creative Commons Attribution License, which permits unrestricted use, distribution, and reproduction in any medium, provided the original work is properly cited.

\begin{abstract}
The aim of this paper is to investigate the dynamical behavior of the following model which describes the logistic difference equation taking into account the subjectivity in the state variables and in the parameters. $x_{n+1}=A x_{n}\left(\tilde{1}-x_{n}\right), n=0,1,2, \cdots$, where $\left\{x_{n}\right\}$ is a sequence of positive fuzzy numbers. $A, \widetilde{1}$ and the initial value $x_{0}$ are positive fuzzy numbers. The existence and uniqueness of the positive solution and global asymptotic behavior of all positive solution of the fuzzy logistic difference equation are obtained. Moreover, some numerical examples are presented to show the effectiveness of results obtained.
\end{abstract}

\section{Introduction}

The first models for growing population were the classical Malthus and Verhulst (or logistic) models which deal with populations with one species [1]. In these models, the identification of the parameters is usually based on statistical methods, starting from data experimentally obtained and on the choice of some method adapted to the identification. These models are often subjected to inaccuracies (fuzzy uncertainty) that can be caused by the nature of the state variables, by parameters as coefficients of the model and by initial conditions.

In our real life, scientists have accepted the fact that uncertainty is very important study in most applications and they also have learned how to deal with uncertainty. Modeling the real life problems in such cases usually involves vagueness or uncertainty in some of the parameters or initial conditions. It is well known that fuzzy set introduced by Zadeh [2] is one of suitable tools and its development has been growing rapidly to various situations of theory and application including the theory of differential equations and difference equations with uncertainty. The latter is known as fuzzy difference equation whose parameters or the initial values are fuzzy numbers, and its solution is a sequence of fuzzy numbers. It has been used to model a dynamical system under possibility uncertainty [3].

To the best of our knowledge, the behavior of the parametric fuzzy difference equation is not always the same as the behavior of corresponding parametric ordinary difference equation. In recent decades, there is an increasing interest in studying fuzzy difference equation by many scholars. Some results concerning the study of fuzzy difference equations are included in these papers (see, for example, [318]).

What we propose in this paper is, to some extent, a generalization of classical logistic discrete model, using the subjectivity which comes from "fuzziness" of the biological phenomenon. The main aim in this paper is to investigate the dynamical behavior of the following logistic discrete time system:

$$
x_{n+1}=A x_{n}\left(\widetilde{1}-x_{n}\right), \quad n=0,1, \cdots,
$$

where parameter $A, \widetilde{1}$ and the initial condition $x_{0}$ are positive fuzzy numbers.

The rest of this paper is organized as follows. In Section 2, we introduce some definitions and preliminaries. In Section 3, we study the existence, uniqueness, and global asymptotic behavior of the positive fuzzy solutions to system 
(1). Some numerical examples are given to show effectiveness of results obtained in Section 4. A general conclusion is drawn in Section 5.

\section{Mathematical Preliminaries}

To be convenience, we give some definitions used in the sequel.

Definition 1 (see [3]). $A$ is said to be a fuzzy number if $A$ : $R \longrightarrow[0,1]$ satisfies (i)-(iv).

1.

(i) $A$ is normal; i.e., there exists an $x \in R$ such that $A(x)=$

(ii) $A$ is fuzzy convex; i.e., for all $t \in[0,1]$ and $x_{1}, x_{2} \in R$ such that

$$
A\left(t x_{1}+(1-t) x_{2}\right) \geq \min \left\{A\left(x_{1}\right), A\left(x_{2}\right)\right\} .
$$

(iii) $A$ is upper semicontinuous.

(iv) The support of $A, \operatorname{supp} A=\overline{\bigcup_{\alpha \in(0,1]}[A]^{\alpha}}=$ $\overline{\{x: A(x)>0\}}$ is compact, where $\bar{A}$ denotes the closure of A.

Let $E^{1}$ be the set of all real fuzzy numbers which are normal, upper semicontinuous, convex, and compactly supported fuzzy sets.

Definition 2 (fuzzy number (parametric form) [19]). A fuzzy number $u$ in a parametric form is a pair $(\underline{u}, \bar{u})$ of functions $\underline{u}(r), \bar{u}(r), 0 \leq r \leq 1$, which satisfies the following requirements:

(1) $\underline{u}(r)$ is a bounded monotonic increasing left continuous function.

(2) $\bar{u}(r)$ is a bounded monotonic decreasing left continuous function.

(3) $\underline{u}(r) \leq \bar{u}(r), 0 \leq r \leq 1$.

A crisp (real) number $x$ is simply represented by $\left(\underline{u}(r), u_{2}(r)\right)=(x, x), 0 \leq r \leq 1$. The fuzzy number space $\{(\underline{u}(r), \bar{u}(r))\}$ becomes a convex cone $E^{1}$ which could be embedded isomorphically and isometrically into a Banach space [17].

Definition 3 (see [19]). Let $u=(\underline{u}(r), \bar{u}(r)), v=(\underline{v}(r), \bar{v}(r)) \in$ $E^{1}, 0 \leq r \leq 1$, and arbitrary $k \in R$. Then

(i) $u=v$ iff $\underline{u}(r)=\underline{v}(r), \bar{u}(r)=\bar{v}(r)$

(ii) $u+v=(\underline{u}(r)+\underline{v}(r), \bar{u}(r)+\bar{v}(r))$

(iii) $u-v=(\underline{u}(r)-\bar{v}(r), \bar{u}(r)-\underline{v}(r))$

(iv)

$$
k u= \begin{cases}(k \underline{u}(r), k \bar{u}(r)), & k \geq 0 ; \\ (k \bar{u}(r), k \underline{u}(r)), & k<0,\end{cases}
$$

$(\mathrm{v}) u v=(\min \{\underline{u}(r) \underline{v}(r), \underline{u}(r) \bar{v}(r), \bar{u}(r) \underline{v}(r), \bar{u}(r) \bar{v}(r)\}$, $\max \{\underline{u}(r) \underline{v}(r), \underline{u}(r) \bar{v}(r), \overline{\bar{u}}(r) \underline{v}(r), \overline{\bar{u}}(r) \bar{v}(r)\})$
Definition 4 (triangular fuzzy number [19]). A triangular fuzzy number (TFN) denoted by $A$ is defined as $(b, c, d)$ where the membership function

$$
A(x)= \begin{cases}0, & x \leq b ; \\ \frac{x-b}{c-b}, & b \leq x \leq c ; \\ 1, & x=c ; \\ \frac{d-x}{d-c}, & c \leq x \leq d ; \\ 0, & x \geq d .\end{cases}
$$

The $\alpha$-cuts of $A=(b, c, d)$ are denoted by $[A]^{\alpha}=\{x \in$ $R: A(x) \geq \alpha\}=[b+\alpha(c-b), d-\alpha(d-c)]=\left[A_{l, \alpha}, A_{r, \alpha}\right]$, $\alpha \in[0,1]$; it is clear that $[A]^{\alpha}$ is a closed interval. A fuzzy number is positive if supp $A \subset(0, \infty)$.

The following proposition is fundamental since it characterizes a fuzzy set through the $\alpha$-levels.

Proposition 5 (see [19]). If $\left\{A^{\alpha}: \alpha \in[0,1]\right\}$ is a compact, convex, and not empty subset family of $R^{n}$ such that

(i) $\overline{\cup A^{\alpha}} \subset A^{0}$

(ii) $A^{\alpha_{2}} \subset A^{\alpha_{1}}$ if $\alpha_{1} \leq \alpha_{2}$

(iii) $A^{\alpha}=\bigcap_{k \geq 1} A^{\alpha_{k}}$ if $\alpha_{k} \uparrow \alpha>0$

then there is $u \in E^{n}$ such that $[u]^{\alpha}=A^{\alpha}$ for all $\alpha \in(0,1]$ and $[u]^{0}=\overline{\bigcup_{0<\alpha \leq 1} A^{\alpha}} \subset A^{0}$.

Definition 6 (see [4]). A sequence $\left\{x_{n}\right\}$ of positive fuzzy numbers persists (resp., is bounded) if there exists a positive real number $M$ (resp., $N$ ) such that

$$
\begin{aligned}
& \operatorname{supp} x_{n} \subset[M, \infty) \\
& \left(\operatorname{resp} . \operatorname{supp} x_{n} \subset(0, N]\right), \\
& \qquad n=1,2, \cdots .
\end{aligned}
$$

A sequence $\left\{x_{n}\right\}$ of positive fuzzy numbers is bounded and persists if there exist positive real numbers $M, N>0$ such that

$$
\operatorname{supp} x_{n} \subset[M, N], \quad n=1,2, \cdots \text {. }
$$

Definition 7. $x_{n}$ is called a positive solution of (1), if $\left\{x_{n}\right\}$ is a sequence of positive fuzzy numbers which satisfies (1). The equilibrium of (1) is the solution of the following equation: $x=A x(\tilde{1}-x)$.

Definition 8 (see [3]). Let $A, B$ be fuzzy numbers with $[A]^{\alpha}=$ $\left[A_{l, \alpha}, A_{r, \alpha}\right],[B]^{\alpha}=\left[B_{l, \alpha}, B_{r, \alpha}\right]$; then the metric of $A$ and $B$ is defined as

$$
D(A, B)=\sup _{\alpha \in(0,1]} \max \left\{\left|A_{l, \alpha}-B_{l, \alpha}\right|,\left|A_{r, \alpha}-B_{r, \alpha}\right|\right\} .
$$

Definition 9. Let $\left\{x_{n}\right\}$ be a sequence of positive fuzzy numbers and $x$ is a positive fuzzy number. Suppose that

$$
\left[x_{n}\right]^{\alpha}=\left[L_{n, \alpha}, R_{n, \alpha}\right], \quad \alpha \in(0,1], n=0,1, \cdots,
$$


and

$$
[x]^{\alpha}=\left[L_{\alpha}, R_{\alpha}\right], \quad \alpha \in(0,1] .
$$

The sequence $\left\{x_{n}\right\}$ converges to $x$ with respect to $D$ as $n \longrightarrow$ $\infty$ if $\lim _{n \rightarrow \infty} D\left(x_{n}, x\right)=0$.

Definition 10. Let $x$ be a positive equilibrium of (1). The positive equilibrium $x$ is stable, if, for every $\varepsilon>0$, there exists a $\delta=\delta(\varepsilon)$ such that for every positive solution $x_{n}$ of (1), which satisfies $D\left(x_{0}, x\right) \leq \delta$, we have $D\left(x_{n}, x\right) \leq \varepsilon$ for all $n \geq 0$. The positive equilibrium $x$ is asymptotically stable, if it is stable and every positive solution of (1) converges to the positive equilibrium of (1) with respect to $D$ as $n \longrightarrow \infty$.

\section{Main Results}

First we study the existence of the positive solutions of (1). We need the following lemma.

Lemma 11 (see [20]). Let $f: R^{+} \times R^{+} \longrightarrow R^{+}$be continuous; $A$ and $B$ are fuzzy numbers. Then

$$
[f(A, B)]^{\alpha}=f\left([A]^{\alpha},[B]^{\alpha}\right), \quad \alpha \in(0,1]
$$

Theorem 12. Consider (1) where $A, \widetilde{1}$ are positive fuzzy numbers. Then, for any positive fuzzy numbers $x_{0}$, there exists a unique positive solution $x_{n}$ of (1).

Proof. The proof is similar to Proposition 2.1 [5]. Suppose that there exists a sequence $\left\{x_{n}\right\}$ of fuzzy numbers satisfying (1) with initial condition $x_{0}$. Consider $\alpha$-cuts, $\alpha \in(0,1], n=$ $0,1,2, \cdots$, applying Lemma 11 ; we have

$$
\begin{aligned}
{\left[x_{n+1}\right]^{\alpha}=\left[L_{n+1, \alpha}, R_{n+1, \alpha}\right]=\left[A x_{n}\left(\widetilde{1}-x_{n}\right)\right]^{\alpha} } \\
=[A]^{\alpha}\left[x_{n}\right]^{\alpha}\left([\widetilde{1}]^{\alpha}-\left[x_{n}\right]^{\alpha}\right) \\
=\left[A_{l, \alpha}, A_{r, \alpha}\right] \times\left[L_{n, \alpha}, R_{n, \alpha}\right] \\
\quad \times\left(\left[\widetilde{1}_{l, \alpha}, \widetilde{1}_{r, \alpha}\right]-\left[L_{n, \alpha}, R_{n, \alpha}\right]\right) \\
=\left[A_{l, \alpha} L_{n, \alpha}\left(\widetilde{1}_{l, \alpha}-R_{n, \alpha}\right), A_{r, \alpha} R_{n, \alpha}\left(\widetilde{1}_{r, \alpha}-L_{n, \alpha}\right)\right] .
\end{aligned}
$$

From (11), we can get the following system of ordinary difference equation with parameter $\alpha \in(0,1]$, for $n=$ $0,1,2, \cdots$,

$$
\begin{aligned}
& L_{n+1, \alpha}=A_{l, \alpha} L_{n, \alpha}\left(\widetilde{1}_{l, \alpha}-R_{n, \alpha}\right), \\
& R_{n+1, \alpha}=A_{r, \alpha} R_{n, \alpha}\left(\widetilde{1}_{r, \alpha}-L_{n, \alpha}\right) .
\end{aligned}
$$

Then, for any initial condition $\left(L_{0, \alpha}, R_{0, \alpha}\right), \alpha \in(0,1]$, there exists a unique solution $\left(L_{n, \alpha}, R_{n, \alpha}\right)$.

Now we show that $\left[L_{n, \alpha}, R_{n, \alpha}\right], \alpha \in(0,1]$, determines the solution of (1) with initial value $x_{0}$, where $\left(L_{n, \alpha}, R_{n, \alpha}\right)$ is the solution of system (12) with initial conditions $\left(L_{0, \alpha}, R_{0, \alpha}\right)$, satisfying

$$
\left[x_{n}\right]^{\alpha}=\left[L_{n, \alpha}, R_{n, \alpha}\right], \quad n=0,1,2, \cdots, \alpha \in(0,1] .
$$

Since $A, \tilde{1}, x_{0}$ are positive fuzzy numbers, for $\alpha_{1}, \alpha_{2} \epsilon$ $(0,1], \alpha_{1} \leq \alpha_{2}$, and, from Definition 2 , then we have

$$
\begin{aligned}
& 0<A_{l, \alpha_{1}} \leq A_{l, \alpha_{2}} \leq A_{r, \alpha_{2}} \leq A_{r, \alpha_{1}} \\
& 0<\widetilde{1}_{l, \alpha_{1}} \leq \widetilde{1}_{l, \alpha_{2}} \leq \widetilde{1}_{r, \alpha_{2}} \leq \widetilde{1}_{r, \alpha_{1}} \\
& 0<L_{0, \alpha_{1}} \leq L_{0, \alpha_{2}} \leq R_{0, \alpha_{2}} \leq R_{0, \alpha_{1}}
\end{aligned}
$$

We claim that, for $n=0,1, \cdots$,

$$
0<L_{n, \alpha_{1}} \leq L_{n, \alpha_{2}} \leq R_{n, \alpha_{2}} \leq R_{n, \alpha_{1}}
$$

Inductively, it is clear that (15) is true for $n=0$. Suppose that (15) holds true for $n \leq k, k \in\{1,2, \cdots\}$. Then, from (12), (14), and (15) for $n \leq k$, it follows that

$$
\begin{aligned}
L_{k+1, \alpha_{1}} & =A_{l, \alpha_{1}} L_{k, \alpha_{1}}\left(\tilde{1}_{l, \alpha_{1}}-R_{k, \alpha_{1}}\right) \\
& \leq A_{l, \alpha_{2}} L_{k, \alpha_{2}}\left(\widetilde{1}_{l, \alpha_{2}}-R_{k, \alpha_{2}}\right)=L_{k+1, \alpha_{2}} \\
& \leq A_{r, \alpha_{2}} R_{k, \alpha_{2}}\left(\widetilde{1}_{r, \alpha_{2}}-L_{k, \alpha_{2}}\right)=R_{k+1, \alpha_{2}} \\
& \leq A_{r, \alpha_{1}} R_{k, \alpha_{1}}\left(\widetilde{1}_{r, \alpha_{1}}-L_{k, \alpha_{1}}\right)=R_{k+1, \alpha_{1}}
\end{aligned}
$$

Therefore, (15) is satisfied.

Moreover, for $\forall \alpha \in(0,1]$, it follows from (12) that

$$
\begin{aligned}
& L_{1, \alpha}=A_{l, \alpha} L_{0, \alpha}\left(\widetilde{1}_{l, \alpha}-R_{0, \alpha}\right), \\
& R_{1, \alpha}=A_{r, \alpha} R_{0, \alpha}\left(\widetilde{1}_{r, \alpha}-L_{0, \alpha}\right) .
\end{aligned}
$$

Since $A, x_{0}$ are positive fuzzy numbers, by Definition 2 , then we have that $A_{l, \alpha}, A_{r, \alpha}, L_{0, \alpha}, R_{0, \alpha}$ are left continuous. From (17) we have that $L_{1, \alpha}, R_{1, \alpha}$ are left continuous. By induction we can get that $L_{n, \alpha}, R_{n, \alpha}$ are left continuous.

Next we prove that the support of $x_{n}, \operatorname{supp} x_{n}=$

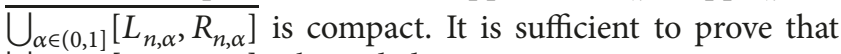
$\bigcup_{\alpha \in(0,1]}\left[L_{n, \alpha}, R_{n, \alpha}\right]$ is bounded.

Let $n=1$; since $A, \widetilde{1}$ and $x_{0}$ are positive fuzzy numbers, there exist constants $M_{A}>0, N_{A}>0, P>0, Q>0, M_{0}>$ $0, N_{0}>0$ such that, for $\alpha \in(0,1]$,

$$
\begin{gathered}
{\left[A_{l, \alpha}, A_{r, \alpha}\right] \subset\left[M_{A}, N_{A}\right],} \\
{\left[\widetilde{1}_{l, \alpha}, \widetilde{1}_{r, \alpha}\right] \subset[P, Q],} \\
{\left[L_{0, \alpha}, L_{0, \alpha}\right] \subset\left[M_{0}, N_{0}\right] .}
\end{gathered}
$$

Hence, from (17) and (18), for $\alpha \in(0,1]$, we get

$$
\left[L_{1, \alpha}, R_{1, \alpha} \subset\left[M_{A} M_{0}\left(P-N_{0}\right), N_{A} N_{0}\left(Q-M_{0}\right)\right] .\right.
$$

It is clear that

$$
\begin{aligned}
& \bigcup_{\alpha \in(0,1]}\left[L_{1, \alpha}, R_{1, \alpha}\right. \\
& \quad \subset\left[M_{A} M_{0}\left(P-N_{0}\right), N_{A} N_{0}\left(Q-M_{0}\right)\right] .
\end{aligned}
$$


Therefore, (20) implies $\overline{\bigcup_{\alpha \in(0,1]}\left[L_{1, \alpha}, R_{1, \alpha}\right]}$ is compact, and $\overline{\bigcup_{\alpha \in(0,1]}\left[L_{1, \alpha}, R_{1, \alpha}\right]} \subset(0, \infty)$. Deducing inductively it can follow easily that

$$
\begin{aligned}
& \overline{\bigcup_{\alpha \in(0,1]}\left[L_{n, \alpha}, R_{n, \alpha}\right]} \text { is compact, } \\
& \text { and } \overline{\bigcup_{\alpha \in(0,1]}\left[L_{n, \alpha}, R_{n, \alpha}\right]} \subset(0, \infty) \text {. }
\end{aligned}
$$

Therefore, from (15), (21), and $L_{n, \alpha}, R_{n, \alpha}$ being left continuous, it can be concluded that $\left[L_{n, \alpha}, R_{n, \alpha}\right]$ determines a sequence $\left\{x_{n}\right\}$ of positive fuzzy numbers satisfied with (13).

We prove that $x_{n}$ is a solution of (11) with initial value $x_{0}$, since, for $\forall \alpha \in(0,1]$,

$$
\begin{aligned}
& {\left[x_{n+1}\right]^{\alpha}=\left[L_{n+1, \alpha}, R_{n+1, \alpha}\right]} \\
& \quad=\left[A_{l, \alpha} L_{n, \alpha}\left(\widetilde{1}_{l, \alpha}-R_{n, \alpha}\right), A_{r, \alpha} R_{n, \alpha}\left(\widetilde{1}_{r, \alpha}-L_{n, \alpha}\right)\right] \\
& \quad=\left[A x_{n}\left(\widetilde{1}-x_{n}\right)\right]^{\alpha} .
\end{aligned}
$$

Namely, $x_{n}$ is a solution of (11) with initial value $x_{0}$.

Suppose that there exists another solution $\bar{x}_{n}$ of (11) with initial value $x_{0}$. Then from arguing as above we can easily get that, for $n=0,1,2, \cdots$,

$$
\left[\bar{x}_{n}\right]^{\alpha}=\left[L_{n, \alpha}, R_{n, \alpha}\right], \quad \alpha \in(0,1] .
$$

Then from (13) and (23), we have $\left[x_{n}\right]^{\alpha}=\left[\bar{x}_{n}\right]^{\alpha}, \alpha \in$ $(0,1], n=0,1,2, \cdots$, and hence $x_{n}=\bar{x}_{n}, n=0,1,2, \cdots$. This completes the proof of Theorem 12 .

In order to study the dynamical behavior of the solution $x_{n}$ to (1), we first consider the following system of difference equations

$$
\begin{aligned}
& y_{n+1}=p y_{n}\left(a-z_{n}\right), \\
& z_{n+1}=q z_{n}\left(b-y_{n}\right), \\
& n=0,1, \cdots .
\end{aligned}
$$

It is clear that the equilibrium points of (24) include the following four cases:

$$
\begin{aligned}
\text { (i) } & (0,0), \\
\text { (ii) } & (0, z), \\
\text { (iii) } & (y, 0), \\
\text { (iv) } & \left(\frac{q b-1}{q}, \frac{p a-1}{p}\right) .
\end{aligned}
$$

Lemma 13. Consider the system of difference equations (24), where $p, q, a$, and $b$ are positive real constants, and the initial values $y_{0}, z_{0}$ are positive real numbers; then the following statements are true.

(i) The equilibrium $(0,0)$ is local asymptotically stable if $p a<1, q b<1$. (ii) System (24) has infinite numbers equilibrium $(0, z)$ which is unstable if $q b=1$.

(iii) System (24) has infinite numbers equilibrium $(y, 0)$ which is unstable if $p a=1$.

(iv) Suppose that $p a>1, q b>1$; then system (24) has a unique positive equilibrium

$$
(\bar{y}, \bar{z})=\left(\frac{q b-1}{q}, \frac{p a-1}{p}\right),
$$

which is unstable.

Proof. (i) It is clear that $(0,0)$ is always equilibrium. We can easily obtain that the linearized system of (24) about the positive equilibrium $(0,0)$ is

$$
\begin{aligned}
& y_{n+1}=p a y_{n}, \\
& z_{n+1}=q b z_{n},
\end{aligned}
$$

$$
n=0,1, \cdots,
$$

from which we can easily obtain that the eigenvalue $\lambda=$ $p a, \lambda=q b$. Since $p a<1$ and $q b<1$, so all of eigenvalue lie inside the unit disk. This implies that the equilibrium $(0,0)$ is local asymptotically stable.

(ii) We can obtain that the linearized system of (24) about the positive equilibrium $(0, z)$ is

$$
\Phi_{n+1}=D \Phi_{n}
$$

where

$$
\begin{aligned}
\Phi_{n} & =\left(\begin{array}{c}
y_{n} \\
z_{n}
\end{array}\right), \\
D & =\left(\begin{array}{cc}
p(a-z) & 0 \\
-q z & q b
\end{array}\right)
\end{aligned}
$$

The character equation of the linearized system of (28) about equilibrium $(0, z)$ is

$$
[\lambda-p(a-z)][\lambda-q b]=0 .
$$

It is clear that the characteristic root of (30) has a root $\lambda=$ $q b=1$. Hence the equilibrium $(0, z)$ is unstable.

(iii) The proof of (iii) is similar to the proof of (ii). So it is omitted.

(iv) We can obtain that the characteristic equation of the linearized system of (28) about equilibrium $(\bar{y}, \bar{z})$ is

$$
\lambda^{2}-2 \lambda+1-(p a-1)(q b-1)=0
$$

The character root of (31) is $\lambda=1 \pm \sqrt{(p a-1)(q b-1)}$. It is clear that there exists a character root larger than 1 . Hence the equilibrium $(\bar{y}, \bar{z})$ is unstable.

Theorem 14. Consider the fuzzy difference equation (1). Suppose that there exists positive constants $N_{A}, Q$, for all $\alpha \in(0,1]$ such that

$$
\begin{gathered}
A_{r, \alpha}<N_{A}, \\
\widetilde{1}_{r, \alpha}<Q, \\
N_{A} Q<1 .
\end{gathered}
$$


Then

$$
\begin{aligned}
{\left[x_{n}\right]^{\alpha} } & =\left[L_{n, \alpha}, R_{n, \alpha}\right], \\
\lim _{n \longrightarrow \infty} D\left(x_{n}, 0\right) & =0 .
\end{aligned}
$$

Proof. Let $x_{n}$ be a positive solution of (1) with initial conditions $x_{0}$; applying (i) of Lemma 13 , we get that $\left(L_{n, \alpha}, R_{n, \alpha}\right), n=$ $0,1, \cdots$, satisfies the following family of systems of parametric ordinary difference equations

$$
\begin{aligned}
& L_{n+1, \alpha}=A_{l, \alpha} L_{n, \alpha}\left(\widetilde{1}_{l, \alpha}-R_{n, \alpha}\right), \\
& R_{n+1, \alpha}=A_{r, \alpha} R_{n, \alpha}\left(\widetilde{1}_{r, \alpha}-L_{n, \alpha}\right),
\end{aligned}
$$

$$
\alpha \in(0,1]
$$

From (34), we have that for all $\alpha \in(0,1]$

$$
\begin{aligned}
& 0<L_{n, \alpha}=A_{l, \alpha} L_{n-1, \alpha}\left(\tilde{1}_{l, \alpha}-R_{n-1, \alpha}\right)<\left(N_{A} Q\right)^{n} L_{0, \alpha}, \\
& 0<R_{n, \alpha}=A_{r, \alpha} R_{n-1, \alpha}\left(\widetilde{1}_{r, \alpha}-L_{n-1, \alpha}\right)<\left(N_{A} Q\right)^{n} R_{0, \alpha}
\end{aligned}
$$

Since $0<N_{A} Q<1$, it follows from (35) that for all $\alpha \in(0,1]$

$$
\begin{aligned}
& \lim _{n \longrightarrow \infty} L_{n, \alpha}=0, \\
& \lim _{n \longrightarrow \infty} R_{n, \alpha}=0 .
\end{aligned}
$$

Therefore, $\lim _{n \rightarrow \infty} D\left(x_{n}, 0\right)=0$.

Theorem 15. Suppose that $A$ and 1 are positive real numbers (trivial fuzzy numbers) and there exists a constant $M>1$ such that

$$
A \geq M
$$

Then (1) has a unique positive equilibrium $x$ such that

$$
\begin{aligned}
& {[x]^{\alpha}=\left[L_{\alpha}, R_{\alpha}\right],} \\
& L_{\alpha}=R_{\alpha}=\frac{A-1}{A}, \\
& \quad \alpha \in(0,1] .
\end{aligned}
$$

Proof. Suppose that there exists a fuzzy number $x$ such that

$$
\begin{aligned}
x & =A x(1-x), \\
{[x]^{\alpha} } & =\left[L_{\alpha}, R_{\alpha}\right], \quad \alpha \in(0,1],
\end{aligned}
$$

where $L_{\alpha}, R_{\alpha} \geq 0$. Then from (39) we can obtain that

$$
\begin{aligned}
& L_{\alpha}=A L_{\alpha}\left(1-R_{\alpha}\right), \\
& R_{\alpha}=A R_{\alpha}\left(1-L_{\alpha}\right) .
\end{aligned}
$$

Hence we can easily obtain that $\left(L_{\alpha}, R_{\alpha}\right)$ is a unique positive solution of (40).

Conversely, we prove that $\left[L_{\alpha}, R_{\alpha}\right], \alpha \in(0,1]$, where $\left(L_{\alpha}, R_{\alpha}\right)$ is the unique positive solution of (40) satisfying (38), determining a fuzzy number $x$ which satisfies (39). From (38) we get, for $\alpha \in(0,1]$,

$$
\begin{aligned}
& L_{\alpha}=A \frac{A-1}{A}\left(1-\frac{A-1}{A}\right) \\
& R_{\alpha}=A \frac{A-1}{A}\left(1-\frac{A-1}{A}\right)
\end{aligned}
$$

Then from (41) for all $\alpha_{1}, \alpha_{2}, 0 \leq \alpha_{1} \leq \alpha_{2} \leq 1$ we have

$$
0<L_{\alpha_{1}} \leq L_{\alpha_{2}} \leq R_{\alpha_{2}} \leq R_{\alpha_{1}} \text {. }
$$

Moreover, since $A$ is a fuzzy number, we have that $A_{l, \alpha}, A_{r, \alpha}$ are left continuous. Then from (37) and (38) we have that $L_{\alpha}, R_{\alpha}$ are left continuous.

Furthermore since (37) holds and $A$ is a positive fuzzy number, there exists a constant $N$ such that $\left[A_{l, \alpha}, A_{r, \alpha}\right] \subset$ $[M, N], \alpha \in(0,1]$. Then from (37) and (38) we obtain that

$$
\left[L_{\alpha}, R_{\alpha}\right] \subset\left[\frac{M-1}{N}, \frac{N-1}{M}\right]
$$

from which we have that

$$
\begin{gathered}
\overline{\bigcup_{\alpha \in(0,1]}\left[L_{\alpha}, R_{\alpha}\right]} \text { is compact, } \\
\overline{\bigcup_{\alpha \in(0,1]}\left[L_{\alpha}, R_{\alpha}\right]} \subset(0, \infty),
\end{gathered}
$$

$$
n=1,2, \cdots,
$$

Therefore, from Theorem 2.1 of [13], (42), (44), and $L_{\alpha}, R_{\alpha}$ being left continuous we have that $x$ is a positive fuzzy number which satisfies (39). This completes the proof of Theorem 15.

Theorem 16. Consider fuzzy difference equation (1); if two positive fuzzy numbers $A, 1$ are all real number 1 (trivial fuzzy numbers) and the initial value $x_{0}$ is a positive fuzzy number, suppose that there exists a positive constant $P, Q, P<Q<1$, such that

$$
\bigcup_{\alpha \in(0,1]}\left[L_{0, \alpha}, R_{0, \alpha}\right] \subset[P, Q] .
$$

Then the solution of system (1) converges to 0.

Proof. By Theorem 12, we have that a sequence of a positive fuzzy number $\left\{x_{n}\right\}$ with $\left[x_{n}\right]^{\alpha}=\left[L_{n, \alpha}, R_{n, \alpha}\right]$ is the solution of (1), satisfying

$$
\begin{aligned}
& L_{n+1, \alpha}=L_{n, \alpha}\left(1-R_{n, \alpha}\right), \\
& R_{n+1, \alpha}=R_{n, \alpha}\left(1-L_{0, \alpha}\right), \\
& n=0,1, \cdots .
\end{aligned}
$$

From (45) and (46), it follows that

$$
\begin{aligned}
& L_{1, \alpha}=L_{0, \alpha}\left(1-R_{0, \alpha}\right)<(1-P) L_{0, \alpha}, \\
& R_{1, \alpha}=R_{0, \alpha}\left(1-L_{0, \alpha}\right)<(1-P) R_{0, \alpha}, \\
& \quad \alpha \in(0,1] .
\end{aligned}
$$


We can prove inductively that

$$
\begin{aligned}
& L_{n, \alpha}<(1-P)^{n} L_{0, \alpha}, \\
& R_{n, \alpha}<(1-P)^{n} R_{0, \alpha}, \\
& \quad n \geq 1, \alpha \in(0,1] .
\end{aligned}
$$

From (46), it is easy to get

$$
\begin{aligned}
& \lim _{n \longrightarrow \infty} L_{n, \alpha}=0, \\
& \lim _{n \longrightarrow \infty} R_{n, \alpha}=0 .
\end{aligned}
$$

Namely, $\lim _{n \rightarrow \infty} D\left(x_{n}, 0\right)=0$.

Remark 17. From Theorem 16, we can conclude that the dynamical behavior of (1) is correlated with the initial value $x_{0}$. When the initial value $x_{0}$ is a positive fuzzy number which is smaller than one, no matter how long the support of $x_{0}$ is, the solution of fuzzy difference equation (1) eventually converges to 0 . From a biological point of view, if the population initial value is too small, even if the growth rate of the population is high (equal one), the population eventually becomes extinct.

\section{Some Illustrative Examples}

In order to illustrate our results obtained, we give some numerical examples to show effectiveness of results.

Example 18. Consider discrete fuzzy logistic system with initial value $x_{0}$ :

$$
x_{n+1}=A x_{n}\left(\tilde{1}-x_{n}\right), \quad n=0,1, \cdots,
$$

where $A, \tilde{1}$ and the initial value $x_{0}$ are positive fuzzy numbers such that

$$
\begin{aligned}
A & =(0.4,0.5,0.6), \\
\widetilde{1} & =(0.5,1,1.5), \\
x_{0} & =(0.3,0.4,0.5)
\end{aligned}
$$

From that, we get, for $\alpha \in(0,1]$,

$$
\begin{aligned}
& {[A]^{\alpha}=[0.4+0.1 \alpha, 0.6-0.1 \alpha],} \\
& {[\widetilde{1}]^{\alpha}=[0.5+0.5 \alpha, 1.5-0.5 \alpha],} \\
& {\left[x_{0}\right]^{\alpha}=\left[0.3+\frac{1}{10} \alpha, 5-\frac{1}{10} \alpha\right] .}
\end{aligned}
$$

From (50) and (52), it results in a coupled system of difference equation with parameter $\alpha \in(0,1]$,

$$
\begin{aligned}
& L_{n+1, \alpha}=(0.4+0.1 \alpha) L_{n, \alpha}\left[0.5+0.5 \alpha-R_{n, \alpha}\right], \\
& R_{n+1, \alpha}=(0.6-0.1 \alpha) R_{n, \alpha}\left[1.5-0.5 \alpha-L_{n, \alpha}\right] .
\end{aligned}
$$

It is clear that (32) of Theorem 14 is satisfied. Therefore, the solution of (50) converges to 0 . (see Figures 1-3)

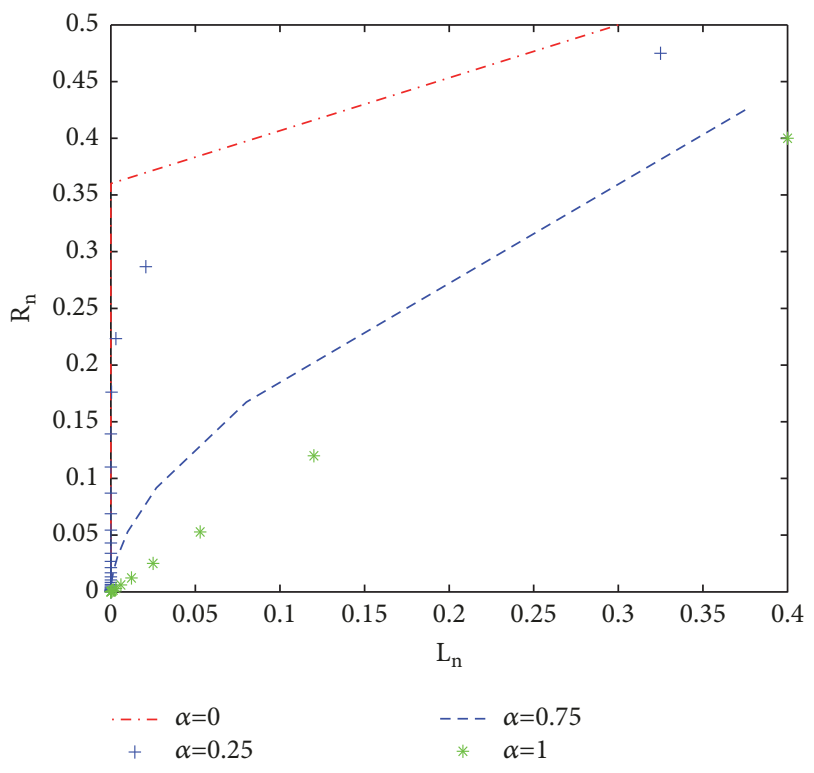

FIgURE 1: The dynamics of system (50) with initial value $x_{0}=$ $(0.3,0.4,0.5)$.
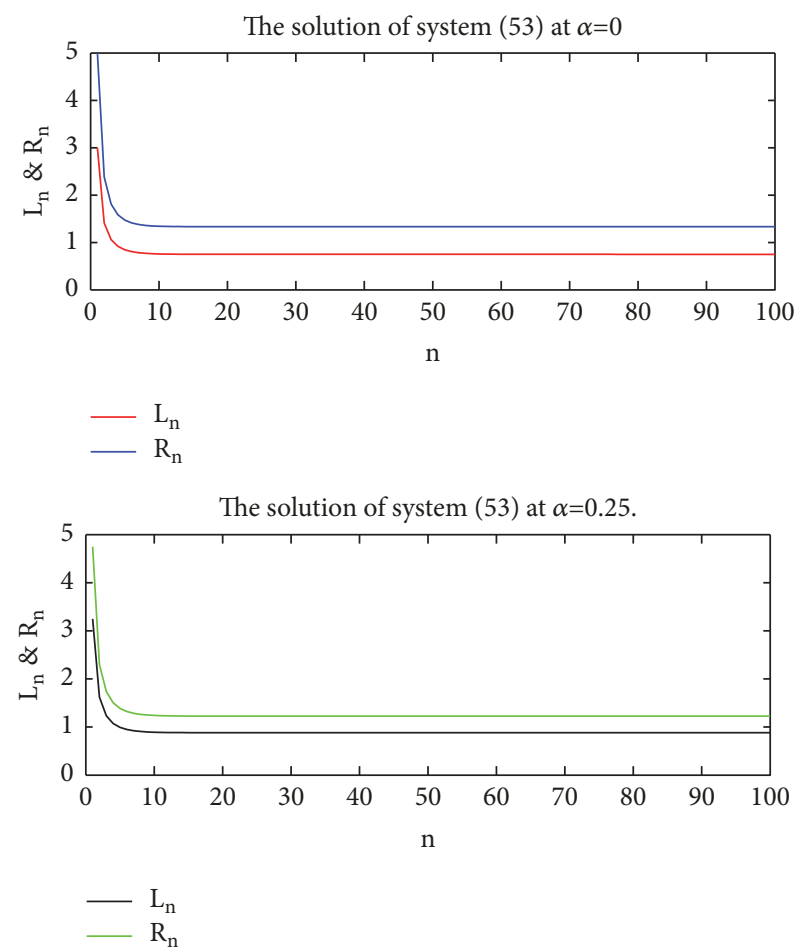

FIGURE 2: The solution of system (53) at $\alpha=0$ and $\alpha=0.25$.

Example 19. Consider discrete fuzzy logistic system (50) with initial value $x_{0}$ where $A=\widetilde{1}=1$ and initial value $x_{0}=$ $(0.7,0.8,0.9)$.

It results in a coupled system of difference equations with parameter $\alpha \in(0,1]$.

$$
\begin{aligned}
& L_{n+1, \alpha}=L_{n, \alpha}\left(1-R_{n, \alpha}\right), \\
& R_{n+1, \alpha}=R_{n, \alpha}\left(1-L_{n, \alpha}\right), \\
& \quad n=0,1,2, \cdots .
\end{aligned}
$$



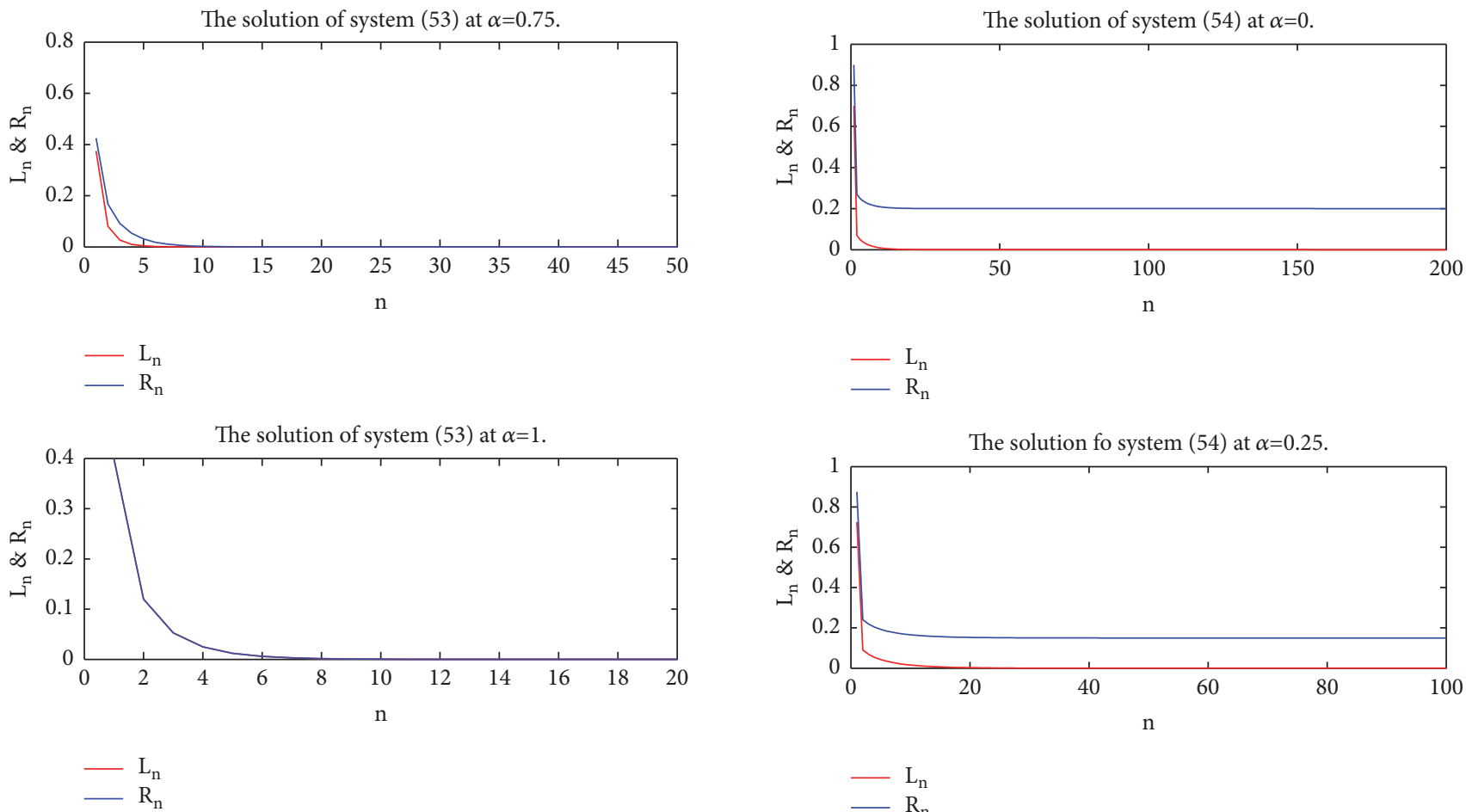

FIgURE 3: The solution of system (53) at $\alpha=0.75$ and $\alpha=1$.
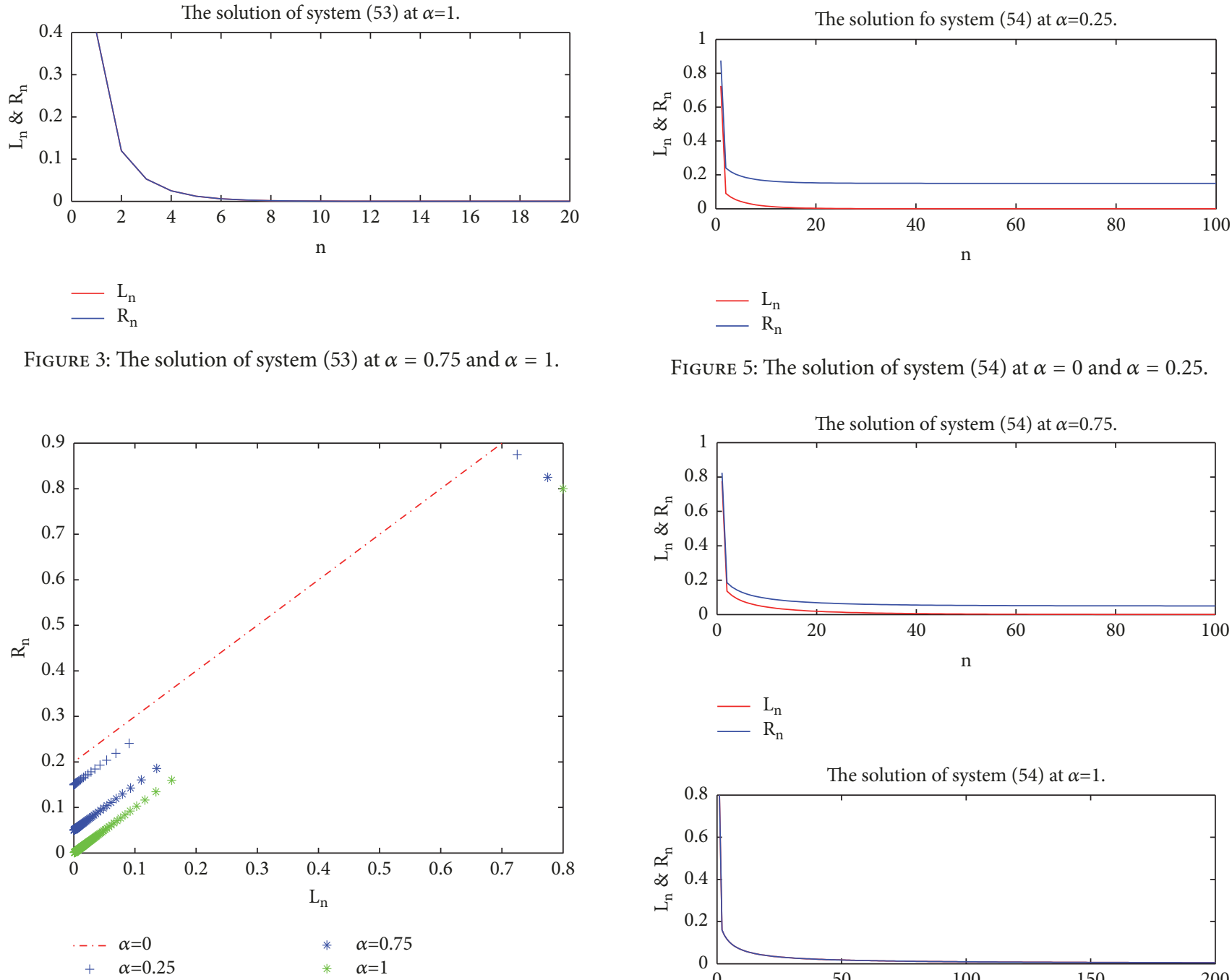

FIGURE 5: The solution of system (54) at $\alpha=0$ and $\alpha=0.25$.
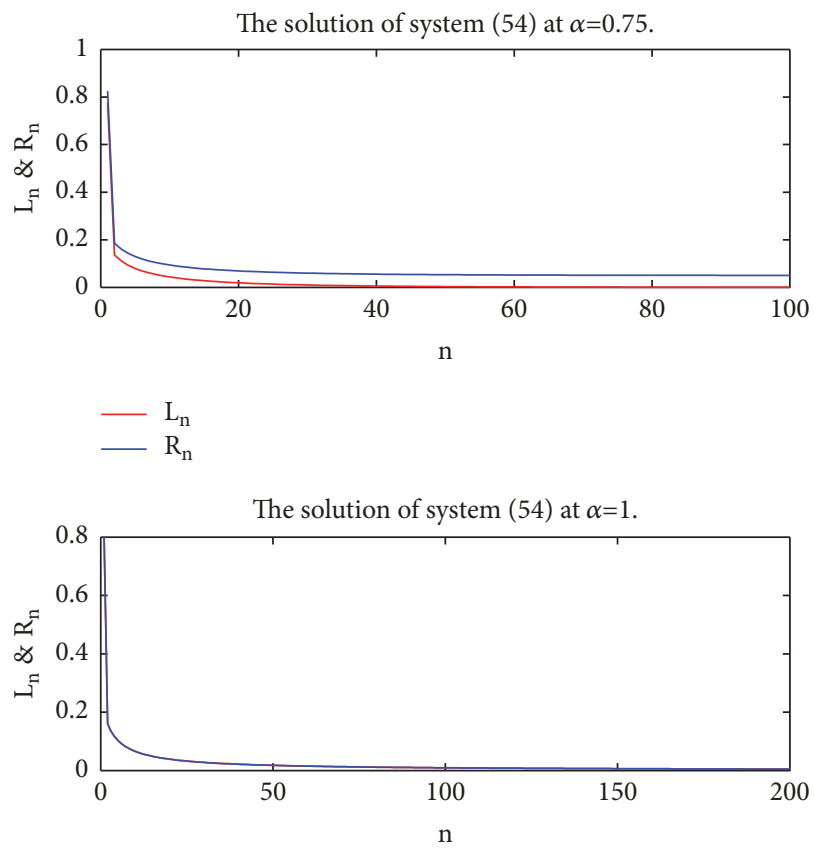

FIgURE 4: The dynamics of system (54) with initial value $x_{0}=$ $(0.7,0.8,0.9)$.

Clearly, all conditions of Theorem 16 are satisfied. Therefore, the solution of (50) converges to 0 (see Figures 4-6).

\section{Conclusion}

This paper deals with the dynamical behavior of single population logistic model under fuzzy environment. Firstly,

the existence of positive fuzzy solution of this model is proved. Secondly, we obtained the following results

(i) If $A_{r, \alpha}<N_{A}, \widetilde{1}_{r, \alpha}<Q, N_{A} Q<1$, then the positive solution of (1) eventually converges to 0 no matter how much the number of population initial values are. 
(ii) If the number of population model is too small, even if the growth rate of population is 1 , the population also converges to 0 (extinct).

(iii) When the parameter of model $A=1$, the system has unique positive equilibrium $x$.

Finally, some examples are presented to show effectiveness of results.

\section{Data Availability}

The data used to support the findings of this study are included within the article.

\section{Conflicts of Interest}

The authors declare that they have no conflicts of interest.

\section{Acknowledgments}

This work was financially supported by the National Natural Science Foundation of China (Grant no. 11761018) and Key Research Project of Guizhou University of Finance and Economics (2018XZD02).

\section{References}

[1] L. Edelstein-Keshet, Mathematical Models in Biology, McGrawHill, Inc., Mexico City, Mexico, 1988.

[2] L. A. Zadeh, "Fuzzy sets," Information and Control, vol. 8, pp. 338-353, 1965.

[3] E. Y. Deeba, A. De Korvin, and E. L. Koh, "A fuzzy difference equation with an application," Journal of Difference Equations and Applications, vol. 2, no. 4, pp. 365-374, 1996.

[4] E. Y. Deeba and A. de Korvin, "Analysis by fuzzy difference equations of a model of $\mathrm{CO}_{2}$ level in the blood," Applied Mathematics Letters, vol. 12, no. 3, pp. 33-40, 1999.

[5] G. Papaschinopoulos and B. K. Papadopoulos, "On the fuzzy difference equation $\mathrm{xn}+1=\mathrm{A}+\mathrm{B} / \mathrm{xn}$," Fuzzy Sets and Systems, vol. 129, no. 1, pp. 73-81, 2002.

[6] G. Papaschinopoulos and B. K. Papadopoulos, "On the fuzzy difference equation $\mathrm{xn}+1=\mathrm{A}+\mathrm{xn} / \mathrm{xn}-\mathrm{m}$," Fuzzy Sets and Systems, vol. 129, no. 1, pp. 73-81, 2002.

[7] G. Stefanidou, G. Papaschinopoulos, and C. J. Schinas, "On an exponential-type fuzzy difference equation," Advances in Difference Equations, vol. 2010, Article ID 196920, pp. 1-19, 2010.

[8] Q. Din, "Asymptotic Behavior of a Second-Order Fuzzy Rational Difference Equation," Journal of Discrete Mathematics, vol. 2015, Article ID 524931, 7 pages, 2015.

[9] R. Memarbashi and A. Ghasemabadi, "Fuzzy difference equations of volterra type," International Journal of Nonlinear Analysis and Applications, vol. 4, pp. 74-78, 2013.

[10] K. A. Chrysafis, B. K. Papadopoulos, and G. Papaschinopoulos, "On the fuzzy difference equations of finance," Fuzzy Sets and Systems, vol. 159, no. 24, pp. 3259-3270, 2008.

[11] Q. H. Zhang, L. H. Yang, and D. X. Liao, "Behavior of solutions to a fuzzy nonlinear difference equation," Iranian Journal of Fuzzy Systems, vol. 9, no. 2, pp. 1-12, 2012.

[12] Q. H. Zhang, L. H. Yang, and D. X. Liao, "On first order fuzzy Ricatti difference equation," Information Sciences, vol. 270, pp. 226-236, 2014.
[13] S. P. Mondal, D. K. Vishwakarma, and A. K. Saha, "Solution of second order linear fuzzy difference equation by Lagrangeïs multiplier method," Journal of Soft Computing and Applications, vol. 1, pp. 11-27, 2016.

[14] Z. Alijani and F. Tchier, "On the fuzzy difference equation of higher order," J. Comput. Complex. Appl, vol. 3, no. 1, pp. 44-49, 2017.

[15] A. Khastan, "Fuzzy logistic difference equation," Iranian Journal of Fuzzy System, vol. 15, no. 7, pp. 55-66, 2018.

[16] C. Wang, X. Su, P. Liu, X. Hu, and R. Li, "On the dynamics of a five-order fuzzy difference equation," Journal of Nonlinear Sciences and Applications. JNSA, vol. 10, no. 6, pp. 3303-3319, 2017.

[17] A. Khastan and Z. Alijani, "On the new solutions to the fuzzy difference equation $\mathrm{xn}+1=\mathrm{A}+\mathrm{B} / \mathrm{xn}$," Fuzzy Sets and Systems, 2018.

[18] A. Khastan, "New solutions for first order linear fuzzy difference equations," Journal of Computational and Applied Mathematics, vol. 312, pp. 156-166, 2017.

[19] D. Dubois and H. Prade, Possibility Theory: An Approach to Computerized Processing of Uncertainty, Plenum Publishing Corporation, New York, NY, USA, 1988.

[20] C. X. Wu and M. Ma, "On embedding problem of fuzzy number space-1," Fuzzy Sets and Systems, vol. 44, no. 1, pp. 33-38, 1991. 


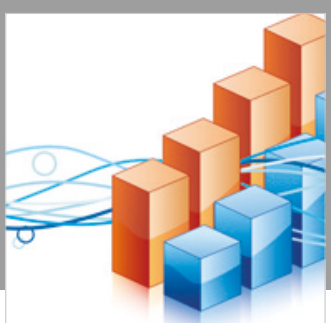

Advances in

Operations Research

\section{-n-m}
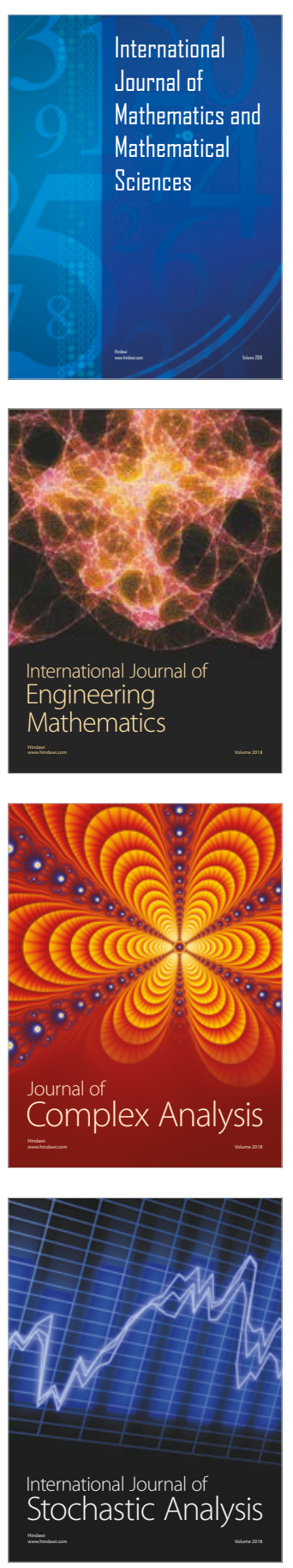
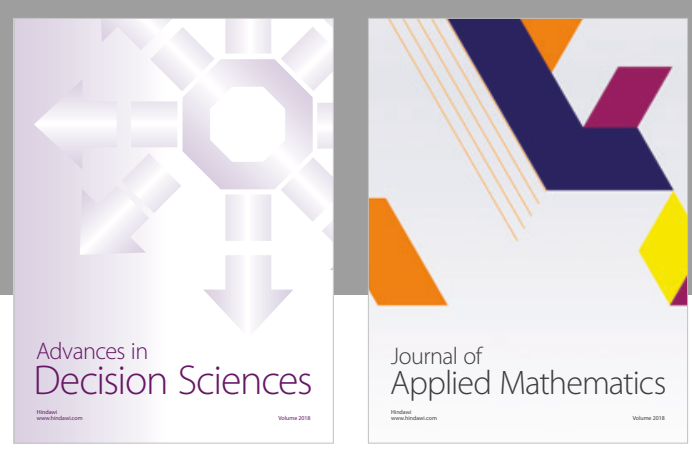

Journal of

Applied Mathematics
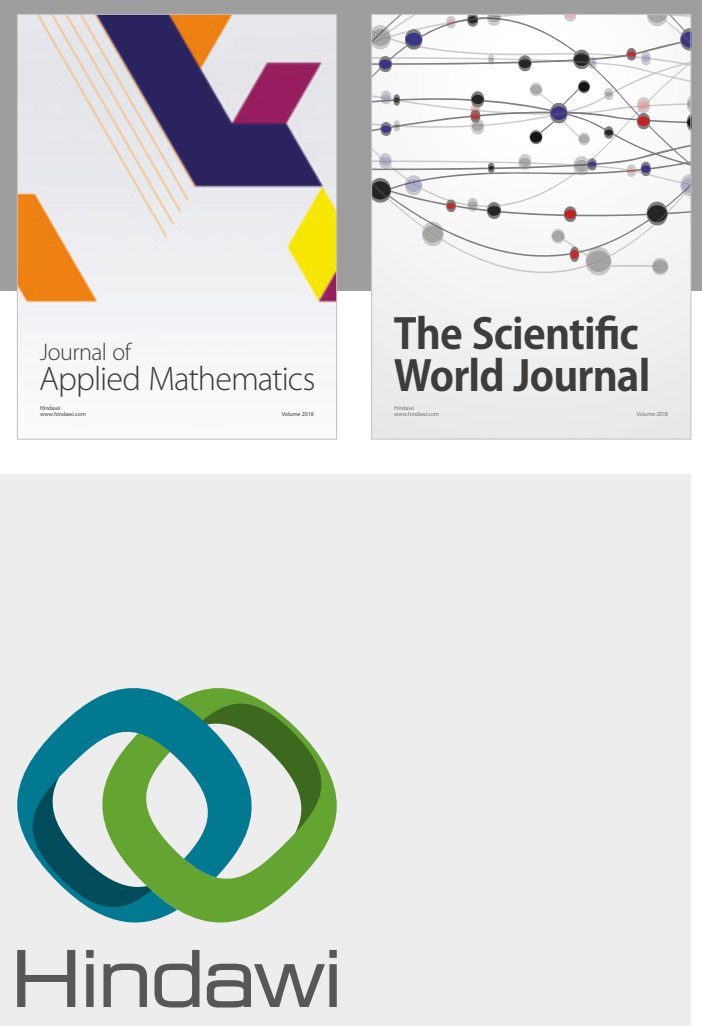

Submit your manuscripts at

www.hindawi.com

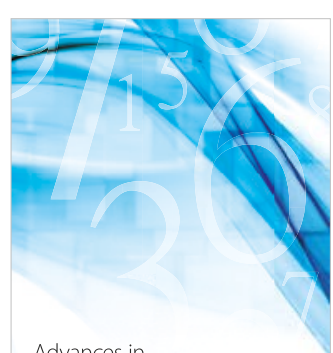

Advances in
Numerical Analysis
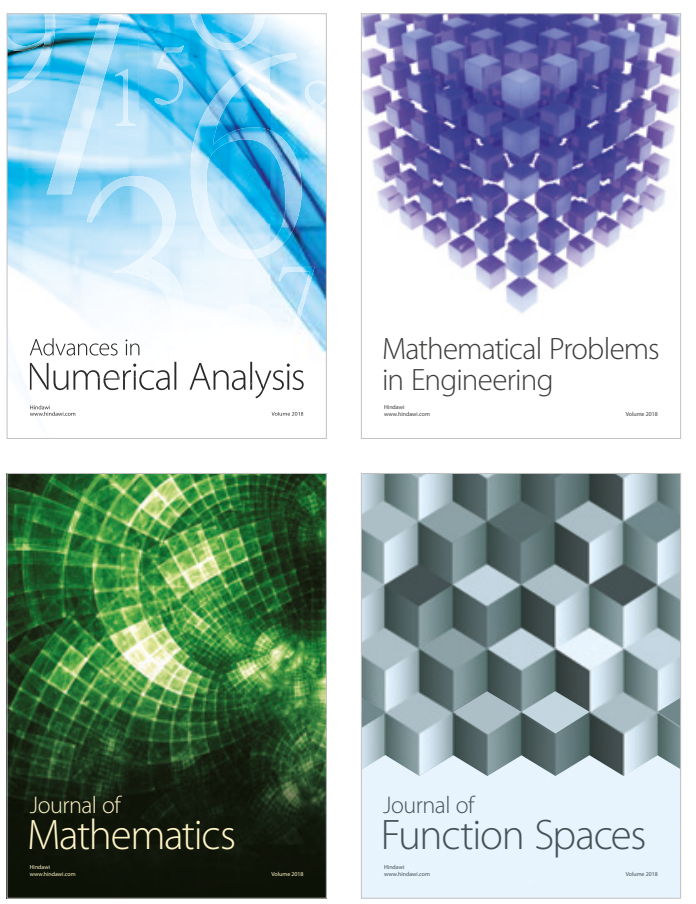

Mathematical Problems in Engineering

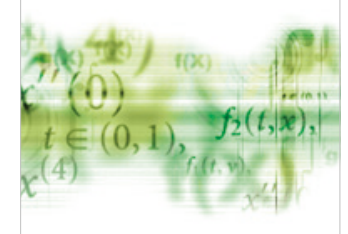

International Journal of

Differential Equations

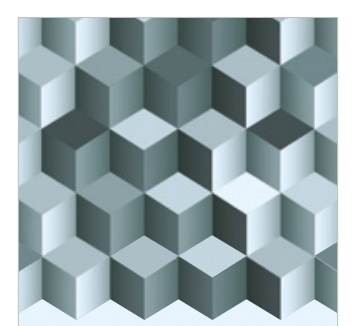

Journal of

Function Spaces

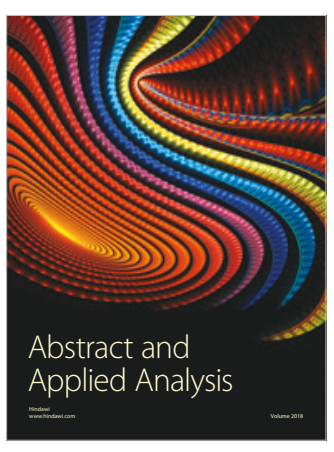

The Scientific

World Journal

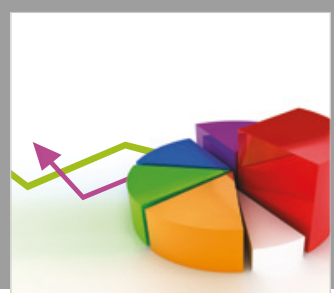

Journal of

Probability and Statistics
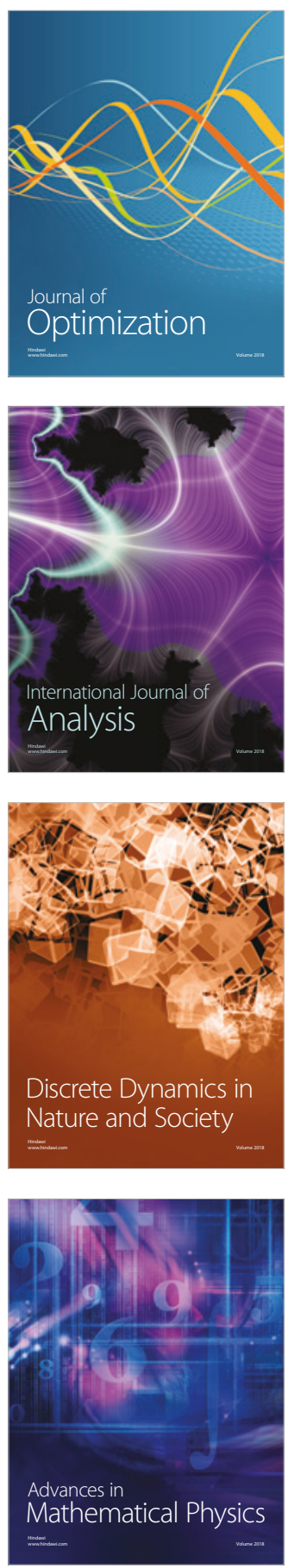